\title{
Icosahedral quasicrystals by optical interference holography
}

\author{
Wing Yim Tam ${ }^{\text {a) }}$ \\ Department of Physics, Hong Kong University of Science and Technology, Clear Water Bay, Kowloon, \\ Hong Kong, China and Institute of Nano Science and Technology, Hong Kong University of Science \\ and Technology, Clear Water Bay, Kowloon, Hong Kong, China
}

(Received 7 September 2006; accepted 10 November 2006; published online 19 December 2006)

\begin{abstract}
Optical interference holography has been proven to be a useful technique in fabricating periodic photonic crystals in which electromagnetic waves are forbidden in certain frequency band gaps. Compared to periodic crystals, quasicrystals, having higher point group symmetry, are more favorable in achieving complete band gaps. In this letter, we propose two seven-beam optical interference configurations based on the reciprocal vector space representation to fabricate icosahedral quasicrystals. Interference simulations for the quasicrystals exhibit the full symmetry of an icosahedron. The result paves the way for the fabrication of photonic quasicrystals using holographic lithography. (c) 2006 American Institute of Physics. [DOI: 10.1063/1.2408651]
\end{abstract}

Quasicrystalline structures (quasicrystals), discovered in alloys in the early 1980s, have higher point group symmetry than ordinary periodic crystals. They exhibit long-range aperiodic order and rotational symmetries that fall outside the traditional crystallographic classification schemes. ${ }^{1}$ It was suggested $^{2}$ that quasicrystals can possess photonic band gaps in which electromagnetic wave propagation is forbidden. ${ }^{3}$ Furthermore, because of the higher rotational symmetry of the quasicrystals, the band gaps are more isotropic and thus are more favorable in achieving complete band gaps than conventional photonic crystals. Photonic crystals have been fabricated by techniques such as the self-assembly of colloidal microspheres or microfabrication, and, recently, holographic lithography and the multiphoton direct laser writing. ${ }^{4-7}$ However, it is difficult to fabricate quasicrystals by the self-assembly and microfabricating techniques in two dimensions (2Ds) and nearly impossible in three dimensions (3Ds). ${ }^{8}$ Using a stereo lithography method, photonic icosahedral quasicrystals have been fabricated exhibiting sizable band gaps in the microwave range. ${ }^{9}$ However, it is still a challenge to fabricate quasicrystals in the visible range. Recently, it has been demonstrated that holographic lithography can be used to fabricate $2 \mathrm{D}$ and quasi-3D quasicrystals in photoresists in the submicron scales. ${ }^{10}$ To create periodic interference pattern for holographic lithography, coherent beams obtained from a single source have to be configured with wave vectors $\left\{\mathbf{k}_{i}\right\}$ such that $\{\Delta \mathbf{k}\}=\left\{\mathbf{k}_{i}-\mathbf{k}_{j}\right\}$ are the reciprocal base vectors $\left\{\mathbf{q}_{i}\right\}$ of the periodic structure. For the usual periodic crystals the reciprocal base vectors are known, e.g., face-centered-cubic lattice can be obtained from body-centered-cubic reciprocal lattice constructed from a four-beam interference with an "inverted-umbrella" configuration. ${ }^{6}$ For quasicrystals, more than four (usually six) reciprocal base vectors are needed, making the task highly nontrivial. Here we report two optical interference configurations for the icosahedral quasicrystal using seven coherent beams from a single light source. The interference patterns obtained from the two configurations display the full symmetry of an icosahedron. More importantly, these beam configurations are accessible in experimental setups commonly used in holographic lithography. Our result paves the

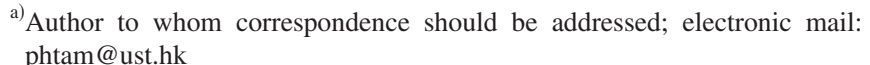

way for the fabrication of photonic quasicrystals in submicron scales using holographic lithography.

Quasicrystals can be classified either as projections of higher-dimensional periodic structures or in terms of wave vectors in the reciprocal space corresponding to the diffraction patterns of the quasicrystals. ${ }^{11}$ It is the reciprocal vector space approach that provides the basis for fabricating quasicrystals using optical interference holography. For the icosahedral lattice, it was shown that there are three distinct reciprocal icosahedral bases: primitive $P^{*}$, face centered $F^{*}$, and body centered $I^{*}$, corresponding to the conventional $P, I$, and $F$ real-space lattices ${ }^{11,12}$ spanned by the linear combinations of six base vectors $\left\{\mathbf{a}_{i}\right\}$ (shown in Table I) with lattice coordinates given by $\mathbf{R}=\sum n_{i} \mathbf{a}_{i}$, for integer subset of $\left\{n_{i}\right\}$.

(The asterisk denotes the reciprocal vector space.) Equations (i) and (ii) in Table I are two bases for the icosahedral quasicrystal dictating the six base vectors along the fivefold axes of an icosahedron. In one basis five base vectors are arranged symmetrically about the sixth vector [Fig. 1(a)] while in the other basis all six base vectors are arranged around a threefold axis [Fig. 1(b)]. ${ }^{11,13}$ We have identified two sets, lattices $A$ (Ref. 11) and $B$, (Ref. 12) of reciprocal primitive base vectors $\left\{\mathbf{q}_{i}\right\}$ ( $i=0-5$ and $1-6$ for lattices $A$ and $B$, respectively) for the face-centered $F^{*}$ lattice [equations (iii) and (iv) in Table I] that can be generated by seven interfering wave vectors $\left\{\mathbf{k}_{i}\right\}$ [equations (v) and (vi) in Table I]. Note that not all the $\left|\mathbf{q}_{i}\right|$ have the same magnitude while $\left|\mathbf{k}_{i}\right|$ are all equal, satisfying the condition that the interfering light beams are from a single light source. No such set of wave vectors is found for the primitive $P^{*}$ and body-centered $I^{*}$ lattices. Equations (vii) and (viii) show the relations between the reciprocal base vectors and the wave vectors for the two lattices while Figs. 1(c) and 1(d) show the geometrical arrangements of the reciprocal base vectors (green arrows) and the wave vectors (blue arrows). For lattice $A$, the five wave vectors $\mathbf{k}_{i}, i=1-5$, are equally spaced around and making an angle $\phi=63.4^{\circ}$ with $\mathbf{k}_{0}$ as shown in Fig. 1(e) the projection along the $\mathbf{k}_{0}$ direction (pointing out of the page). Note that $\mathbf{k}_{6}$ is also along the axis of symmetry but points in the opposite direction of $\mathbf{k}_{0}$. As for lattice $B$, the axis of symmetry is also along the $\mathbf{k}_{0}$ direction with three wave vectors $\left(\mathbf{k}_{1}, \mathbf{k}_{2}, \mathbf{k}_{3}\right)$ evenly distributed around and making an angle $\phi=41.8^{\circ}$ with $\mathbf{k}_{0}$. The other three wave vectors $\left(\mathbf{k}_{4}, \mathbf{k}_{5}, \mathbf{k}_{6}\right)$, also 
TABLE I. Lattice base vectors $\{\mathbf{a}\}$, reciprocal primitive base vectors $\{\mathbf{q}\}$, and the wave vectors $\{\mathbf{k}\}$ of the interfering beams from a coherent light source for two lattices of icosahedral quasicrystals. The last row shows the relation between the reciprocal base vectors and the wave vectors of the interfering beams. $\tau=(1+\sqrt{5}) / 2$ is the golden mean.

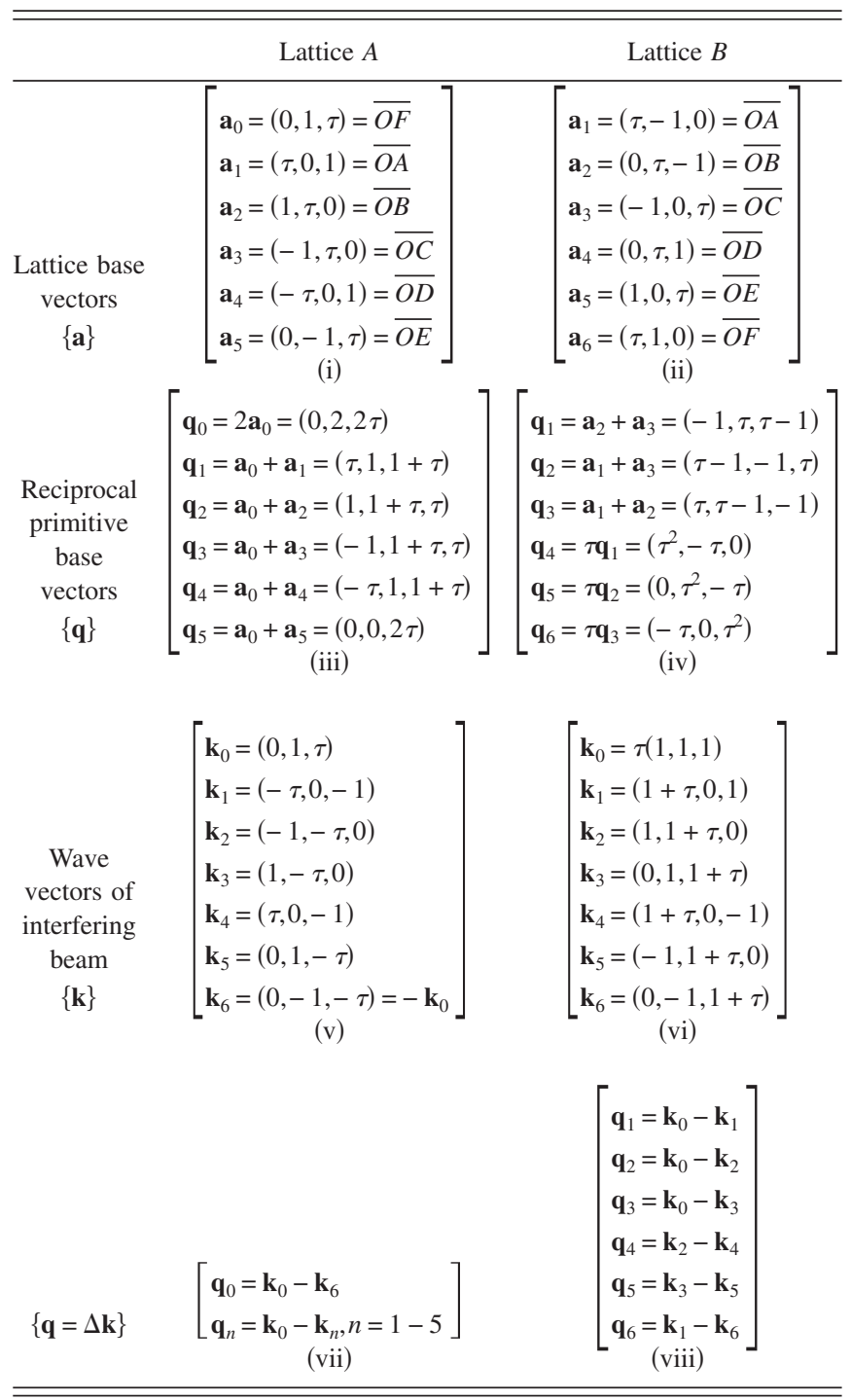

evenly distributed around but making an angle $\phi=70.5^{\circ}$ with $\mathbf{k}_{0}$, are displaced at an angle $37.7^{\circ}$ from the first three wave vectors, as shown in Fig. 1(f). One unique feature for these two lattices is that the wave vectors can be easily achieved by seven beams from a single light source, enabling possible realization of the icosahedral quasicrystals using standard holographic lithography setups. ${ }^{6,14}$

With the wave vectors from equations (v) and (vi), the interference pattern of the seven coherent beams is given by

$$
I(\mathbf{r})=\sum_{l, m} \mathbf{E}_{l} e^{-i \mathbf{k}_{l} \cdot \mathbf{r}-i \delta_{l}} \cdot \mathbf{E}_{m}^{*} e^{i \mathbf{k}_{m} \cdot \mathbf{r}+i \delta_{m}}
$$

where $l, m=0-6 ; \mathbf{E}_{l}$ and $\delta_{l}$ are the electric field and its phase for wave vector $\mathbf{k}_{l}$. We define the polarization of the side beam $\mathbf{k}_{l}$ as the angle $\omega_{l}$ of the electric field $\mathbf{E}_{l}$ from the plane of incident formed by the wave vectors $\mathbf{k}_{l}$ and the central wave vector $\mathbf{k}_{0}$. The polarizations of the central beams $\mathbf{k}_{0}$ and $\mathbf{k}_{6}$ (for lattice $A$ only) are taken as the angle between their projections on the $x y$ plane with the $x$ axis. Given a light source, $k=2 \pi / \lambda$, where $\lambda$ is the wavelength of the

source, the free parameters in the model are $\left|\mathbf{E}_{l}\right|, \omega_{l}$, and $\delta_{l}$. icosahedron construction is equally good.
Downloaded 07 May 2009 to 143.89.106.174. Redistribution subject to AlP license or copyright; see http://aplaip.

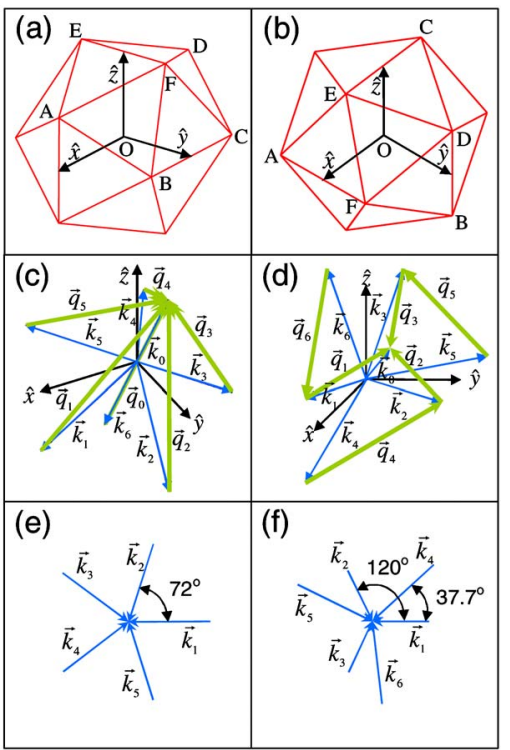

FIG. 1. (Color online) [(a) and (b)] Icosahedral quasicrystals using the lattice base vectors in Equations (i) and (ii) in Table I, respectively. [(c) and (d)] Beam configurations for the wave vectors $\{\mathbf{k}\}$ (blue) in Equations (v) and (vi) and the constructions for the reciprocal base vectors $\{\mathbf{q}\}$ (green) in Equations (iii) and (iv) respectively. [(e) and (f)] Projections of the wave vectors on the planes perpendicular to the $\mathbf{k}_{0}$ direction for the beam configurations in (c) and (d), respectively.

For simplicity, $\left|\mathbf{E}_{l}\right|$ can be taken as the same for all wave vectors, leaving only $\omega_{l}$ and $\delta_{l}$ as adjustable parameters. We determine the polarization of each side beam by requiring maximum contrast for the interference between the individual side beam $\mathbf{k}_{l}$ and the central beam $\mathbf{k}_{0}$. As for the phases, four of them can be arbitrarily set to zero because it is the difference of the phases that determines the final pattern, leaving only three phases as free parameters. In principle more sophisticated methods can be used to select the optimal parameters. Here, we take it as a "proof-ofprinciple" and vary the parameters to study their effects.

Figure 2(b) shows the interference pattern as intensity contour surfaces for lattice $A$ from Eq. (1) using wave vectors given by equation $(\mathrm{v})$ with maximum contrast polarizations $\left\{\omega_{i}\right\}=\left\{0^{\circ}, 0^{\circ},-82^{\circ}, 58^{\circ},-58^{\circ}, 82^{\circ}, 0^{\circ}\right\}$ and equal phases $\left\{\delta_{i}\right\}=\left\{0^{\circ}, 0^{\circ}, 0^{\circ}, 0^{\circ}, 0^{\circ}, 0^{\circ}, 0^{\circ}\right\}$. The $3 \mathrm{D}$ perspective image viewed along the [111] direction in Fig. 2(b) consists of singly connected contour surfaces, shown as "dots," at $60 \%$ intensity cutoff. The threefold symmetry is clearly shown. The other images in Fig. 2(b) are projections along the different symmetry axes as indicated in the icosahedral quasicrystal shown in the figure. The twofold, threefold, and fivefold symmetries of the icosahedron are clearly shown resembling closely to the corresponding projections in Fig. 2(a) for an icosahedron obtained by placing "atoms" at lattice sites $\mathbf{R}$ with $\left\{n_{i}=0, \pm 1, \pm 2\right\}$. Note that the construction projections will look denser for larger $n_{i}$. Nevertheless, the simulation and the construction agree very well. Note that for lower intensity cutoffs, the dots will become interconnected and form doubly connected contour surfaces as required for recording in photoresists. Figure 2(c) is a simulation with $60 \%$ intensity cutoff for lattice $B$ using equation (vi) with $\left\{\omega_{i}\right\}=\left\{0^{\circ}, 0^{\circ}, 67^{\circ},-67^{\circ}\right.$, $\left.-67^{\circ}, 51^{\circ}, 87^{\circ}\right\}$ and equal phases. The agreement with the icosahedron construction is equally good.

to AIP license or copyright; see http://apl.aip.org/apl/copyright.jsp 

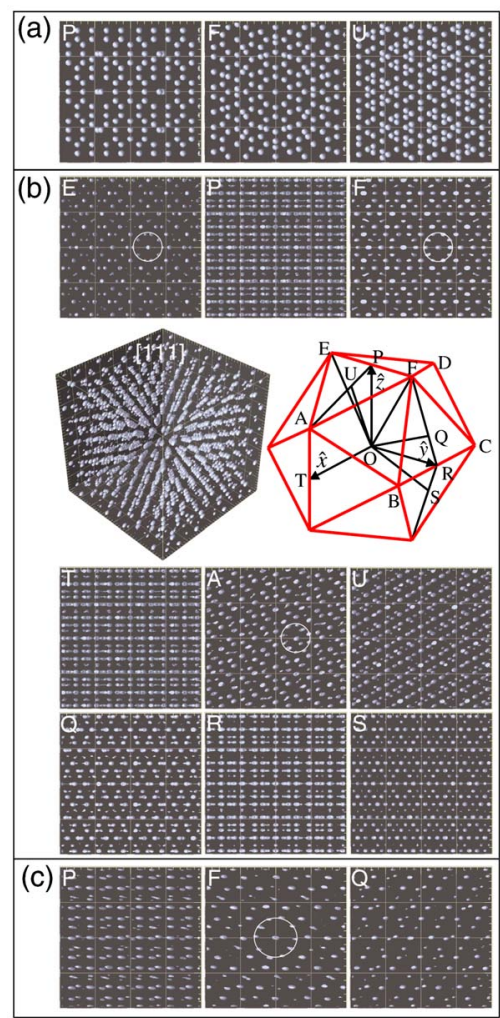

FIG. 2. (Color online) (a) Projections of the icosahedral quasicrystal constructed by attaching spherical balls to lattice sites spanned by $\mathbf{R}$ using Equation (i) in Table I. [(b) and (c)] Projections of icosahedral quasicrystals, displayed in contour surfaces with a $60 \%$ intensity cutoff, constructed by the beam configurations in Figs. 1(c) and 1(d), respectively. The 3D lattice (red) shows the projection directions. The 3D image in (b) shows the perspective view along the [111] direction. The circles drawn in the projections are guides for the five-fold symmetry. The beams used have the same phases with the polarizations for (b) $\left\{\omega_{i}\right\}=\left\{0^{\circ}, 0^{\circ},-82^{\circ}, 58^{\circ},-58^{\circ}, 82^{\circ}, 0^{\circ}\right\}$ and (c) $\left\{\omega_{i}\right\}=\left\{0^{\circ}, 0^{\circ}, 67^{\circ},-67^{\circ},-67^{\circ}, 51^{\circ}, 87^{\circ}\right\}$.

While the full icosahedral symmetry is obtained using the maximum contrast configurations, it turns out that the interference pattern is sensitive to the polarizations of the beams as shown in Fig. 3(a), a simulation for lattice $A$ using $\left\{\omega_{i}\right\}=\left\{0^{\circ}, 0^{\circ}, 0^{\circ}, 0^{\circ}, 0^{\circ}, 0^{\circ}, 0^{\circ}\right\}$ and $\left\{\delta_{i}\right\}$ $=\left\{0^{\circ}, 0^{\circ}, 0^{\circ}, 0^{\circ}, 0^{\circ}, 0^{\circ}, 0^{\circ}\right\}$. Except the fivefold symmetry along the axis of symmetry of the interfering beams, the two-fold and three-fold symmetries are lost as shown in the $P$ (two-fold) and $Q$ (three-fold) projections, demonstrating that this set of polarizations does not produce the full symmetry of the icosahedral quasicrystal. In contrast to the polarizations, the phases of the beams do not seem to be critical as shown in Fig. 3(b), a simulation with arbitrary phases but keeping the polarizations as used in Fig. 2(b). The full sym-

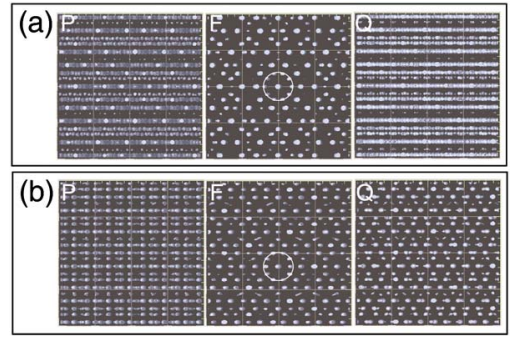

FIG. 3. Projections of icosahedral quasicrystals constructed using the beam configurations in Fig. 1(b) using (a) $\left\{\omega_{i}\right\}=\left\{0^{\circ}, 0^{\circ}, 0^{\circ}, 0^{\circ}, 0^{\circ}, 0^{\circ}, 0^{\circ}\right\}$, and (b) $\left\{\omega_{i}\right\}=\left\{0^{\circ}, 0^{\circ},-82^{\circ}, 58^{\circ},-58^{\circ}, 82^{\circ}, 0^{\circ}\right\}$ and $\left\{\delta_{i}\right\}$ $=\left\{0^{\circ}, 37^{\circ}, 68^{\circ}, 36^{\circ}, 90^{\circ}, 26^{\circ}, 47^{\circ}\right\}$. metry for the icosahedron is still discernible except that the patterns are shifted as compared to those shown in Fig. 2(b). Similar results are obtained for more than 100 simulations with random phases. This insensitiveness to the phases of the interfering beams increases the success rate of fabricating the icosahedral quasicrystal using the holographic lithography technique, which has been implemented recently in an experiment to fabricate icosahedral quasicrystals for the visible range. ${ }^{14}$

To conclude, we have identified two sets of reciprocal base vectors in the face-centered $F^{*}$ lattice representation for icosahedral quasicrystals such that the reciprocal vectors can be obtained from seven wave vectors with the same wavelength. The interference patterns obtained from the seven beams with the correct parameters display the full symmetry of an icosahedron. More importantly, the beam configurations are easily accessible to experiment using simple optical interference setups. This work provides the foundation for a recent experiment in the fabrication of photonic quasicrystals for the visible range using holographic lithography. ${ }^{14}$

Support from Hong Kong RGC Grant No. CA02/ 03.SC01, HKUST603303, and HKUST603405 is gratefully acknowledged. The authors thank Jeffrey C. W. Lee, C. T. Chan, and N. Wang for helpful discussions.

${ }^{1}$ See The Physics of Quasicrystals, edited by P. J. Steinhardt and S. Ostlund (World Scientific, Singapore, 1987); Physical Properties of Quasicrystals, edited by Z. M. Stadnik (Springer, New York, 1999).

${ }^{2}$ Y. S. Chan, C. T. Chan, and Z. Y. Liu, Phys. Rev. Lett. 80, 956 (1998); X. Zhang, Z. Q. Zhang, and C. T. Chan, Phys. Rev. B 63, 081105/1 (2001). ${ }^{3}$ See, e.g., Photonic Band Gap materials, edited by C. M. Soukoulis (Kluwer, Dordrecht, 1996); E. Yablonovitch, Phys. Rev. Lett. 58, 2059 (1987); S. John, ibid. 58, 2486 (1987).

${ }^{4}$ See, e.g., A. Chutinan and S. Noda, Phys. Rev. B 57, R2006 (1998); O. Toader and S. John, Science 292, 1133 (2001); See, e.g., N. Yamamoto, S. Noda, and A. Sasaki, Jpn. J. Appl. Phys., Part 1 36, 1907 (1997); S. Y. Lin, J. G. Fleming, D. L. Hetherington, B. K. Smith, R. Biswas, K. M. Ho, M. M. Sigalas, W. Zubrzycki, S. R. Kurtz, and J. Bur, Nature (London) 394, 251 (1998); A. van Blaaderen, Science 282, 887 (1998).

${ }^{5}$ See, e.g., J. E. G. J. Wijnhoven and W. L. Vos, Science 281, 802 (1998); E. Palacios-Lidón, A. Blanco, M. Ibisate, F. Meseguer, C. López, and J. Sánchez-Dehesa, Appl. Phys. Lett. 81, 4925 (2002); W. Li, G. Sun, F. Tang, W. Y. Tam, J. Li, C. T. Chan, and P. Sheng, J. Phys.: Condens. Matter 17, 2177 (2005).

${ }^{6}$ M. Campbell, D. N. Sharp, M. T. Harrison, R. G. Denning, and A. J. Turberfield, Nature (London) 404, 53 (2000); S. Yang, M. Megens, J. Aizenberg, P. Wiltzius, P. M. Chaikin, and W. B. Russel, Chem. Mater. 14, 2831 (2002); Yu. V. Miklyaev, D. C. Meisel, A. Blanco, G. von Freymann, K. Busch, W. Koch, C. Enkrich, M. Deubel, and M. Wegener, Appl. Phys. Lett. 82, 1284 (2003); X. Wang, J. F. Xu, H. M. Su, Z. H. Zeng, Y. L. Chen, H. Z. Wang, Y. K. Pang, and W. Y. Tam, ibid. 82, 2212 (2003).

${ }^{7}$ M. Deuble, G. Von Freymann, M. Wegener, S. Pereira, K. Busch, and C. M. Soukoulis, Nat. Mater. 3, 444 (2004); M. Deubel, M. Wegener, A. Kaso, and S. John, Appl. Phys. Lett. 85, 1895 (2004).

${ }^{8}$ See, e.g., M. E. Zoorob, M. D. B. Charlton, G. J. Parker, J. J. Baumberg, and M. C. Netti, Nature (London) 404, 740 (2000); M. A. Kaliteevski, S. Brand, R. A. Abram, T. F. Krauss, P. Millar, and R. M. De La Rue, J. Phys.: Condens. Matter 13, 10459 (2001).

${ }^{9}$ W. Man, M. Megens, P. J. Steinhardt, and P. M. Chaikin, Nature (London) 436, 993 (2005).

${ }^{10}$ X. Wang, C. Y. Ng, W. Y. Tam, C. T. Chan, and P. Sheng, Adv. Mater. (Weinheim, Ger.) 15, 1526 (2003); X. Wang, J. Xu, J. C. W. Lee, Y. K. Pang, W. Y. Tam, C. T. Chan, and P. Sheng Appl. Phys. Lett. 88, 051901 (2006).

${ }^{11}$ D. S. Rokhsar, D. C. Wright, and N. D. Mermin, Phys. Rev. B 37, 8145 (1988).

${ }^{12}$ D. S. Rokhsar, N. D. Mermin, and D. C. Wright, Phys. Rev. B 35, 5487 (1987).

${ }^{13}$ N. D. Mermin, Rev. Mod. Phys. 64, 3 (1992).

${ }^{14}$ J. Xu, R. Ma, Xia Wang, and W. Y. Tam (private communication). 